\title{
EL ECOSISTEMA PROGRAMÁTICO. LA NUEVA PUBLICIDAD DIGITAL QUE CONECTA DATOS CON PERSONAS
}

\author{
The programatic ecosystem. The new digital \\ advertising that connects data with people
}

\section{María-Victoria Carrillo-Durán y Ana Rodríguez-Silgado}

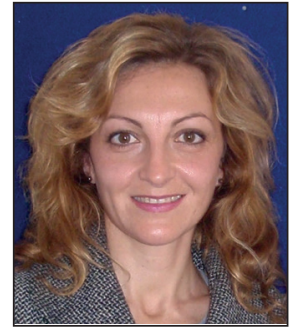

María-Victoria Carrillo-Durán es profesora titular de la Universidad de Extremadura y Master en marketing profesional y gestión publicitaria por la Universidad Complutense de Madrid (UCM). Imparte docencia en los grados de Comunicación audiovisual y de Información y documentación. Su investigación gira en torno a la comunicación online y en el medio real en las organizaciones. Es la investigadora principal del grupo de investigación $A R-C O$, y ha dirigido proyectos de investigación I+D, del Plan Nacional, y del Programa COST (UE). Cuenta con tres sexenios de investigación. http://www.grupoarco.com https://orcid.org/0000-0002-1256-8870

Universidad de Extremadura Facultad de Ciencias de la Documentación y la Comunicación Plaza Ibn Marwan, s/n. 06001 Badajoz, España vicduran@unex.es

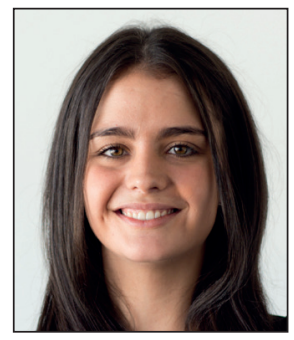

Ana Rodríguez-Silgado es graduada en comunicación audiovisual por la Universidad de Extremadura y titulada en la EUDE Business School en marketing digital. Comenzó como performance marketing specialist en Performics (Grupo Publicis), donde se gestionan campañas de publicidad programática que forman parte de la estrategia global de importantes clientes. Actualmente trabaja para Omnicom Media Group como performance specialist en la agencia de medios OMD. http://www.omd.com https://orcid.org/0000-0003-4384-5591

Omnicom Media Group (OMD) C/ Mesena, 22. 28033 Madrid, España arodriguzs@alumnos.unex.es

\section{Resumen}

Se analiza la publicidad programática como proceso capaz de ofrecer ventajas a las empresas, combinando la gestión de datos de audiencias con la automatización /tecnología y el factor humano. La premisa de partida es que para resolver el problema de la saturación publicitaria hay que conectar los contenidos con los individuos, uno a uno y en tiempo real. El trabajo aúna el conocimiento académico y profesional para analizar las claves de esta nueva forma de publicidad digital y sus principales desafíos. Se incide además en las sinergias entre las ciencias de la información y el futuro de la publicidad programática.

\section{Palabras clave}

Publicidad programática; Publicidad digital; Datos; Gestión de datos; Saturación publicitaria; Personalización; Información; Comunicación; Internet; Perfiles profesionales.

\begin{abstract}
Programmatic advertising as a process capable of offering advantages for companies, combining audience data management with automation/technology and the human factor is analyzed. The starting premise is that in order to solve the problem of advertising saturation, contents has to be connected with the individuals, one by one and in real time. This work brings together the academic and professional knowledge to analyze the keys to this new form of digital advertising and its main challenges. There is also a focus on synergies between the information sciences and the future of programmatic advertising.
\end{abstract}

\section{Keywords}

Programatic advertising; Digital advertising; Data; Data management; Advertising saturation; Personalization; Information; Communication; Internet; Professional profiles. 
Carrillo-Durán, María-Victoria; Rodríguez-Silgado, Ana (2018). “El ecosistema programático. La nueva publicidad digital que conecta datos con personas". El profesional de la información, v. 27, n. 1, pp. 195-201.

https://doi.org/10.3145/epi.2018.ene.18

\section{Introducción}

En el mundo digitalizado actual la información es poder (Gilardoni, 2013). Lo importante no es la cantidad de información, sino su calidad. Gracias a internet las empresas manejan gran cantidad de datos, pero su máxima aspiración no es tenerlos, sino como afirma Serrano-Cobos:

"la cuestión es qué hacer con ellos y cómo" (Serrano-Cobos, 2014, p. 562).

Por ello es fundamental generar datos, organizarlos y compartirlos (Aguado; Martínez-Martínez; Cañete-Sanz, 2015), pero sobre todo, lo más importante es convertirlos en información útil para usarlos en beneficio económico. En la gestión de datos existen varios problemas por resolver, siendo el más importante saber cómo encontrar la información precisa en tiempo real (Serrano-Cobos, 2014) y cómo generar conocimiento práctico comprensible por quienes tienen que tomar las decisiones.

Este reto de la gestión de los datos ha sido planteado desde distintas áreas del entorno audiovisual (Fernández-Manzano; Neira; Clares-Gavilán, 2016); de comunicación en los medios sociales (Martínez-Martínez; Lara-Navarra, 2015); en móviles (Aguado; Martínez-Martínez; Cañete-Sanz, 2015), y en la publicidad (Selva-Ruiz; Caro-Castaño, 2016).

No parece claro cómo dar ese paso último hacia la utilidad, dentro de cada campo de aplicación. Como explican Selva-Ruiz y Caro-Castaño (2016) en el entorno de la publicidad, las posibilidades que pueden ofrecer los datos están aún por explotar en su aplicación a la personalización de los anuncios individuo a individuo y a la inmediatez. Martínez-Martínez y Lara-Navarra (2015) plantean también la necesidad de aportar soluciones para abundar en la precisión de los datos y garantizar la eficacia de la publicidad, mermada por la saturación de anuncios (Martínez-Martínez; Aguado; Boeykens, 2017).

En esta línea, la publicidad programática trata de acortar la distancia entre el dato y su utilidad real. Este trabajo analiza el ecosistema programático buscando las sinergias entre las ciencias de la información y el futuro de la comunicación publicitaria (Bahamonde, 2016). Se identifican sus factores clave y los retos que garantizarán su evolución.

Para abordar este estudio se ha realizado un análisis cross-fertilization que equilibra la vertiente académica y la profesional. Se han utilizado fuentes secundarias, tales como documentos publicados desde el entorno profesional, y también trabajos de investigación académicos.

El artículo parte de las conclusiones de un panel Delphi realizado para aclarar el fenómeno de la publicidad programática (Rodríguez-Silgado, 2017). El método Delphi pretendió definir y ubicar la publicidad programática en el contexto digital. En este trabajo se aprovecha la interacción con estos profesionales para analizar los retos y oportunidades futuras.
Los expertos consultados pertenecían al departamento de publicidad programática del grupo Publicis Media, pertenecientes a los equipos Trading Desk de Vivaki (audience on demand). Además se contó con la colaboración del Club de Expertos en Publicidad Programática, organizadores del / y II Foro de publicidad programática (2015 y 2016).

El resultado fue un panel de veinticuatro expertos de diferentes perfiles, que se pueden agrupar en traders, performance planners, programmatic sales, y data \& technology directors. Al margen del panel Delphi, aportaron información clave para entender los últimos cambios y retos de este nuevo ecosistema publicitario.

\section{De la publicidad online a la publicidad programática. La premisa de partida}

La publicidad programática es algo novedoso y diferente a la publicidad online tradicional (tabla 1), sobre la que aún no existe mucha bibliografía académica, destacando algunas referencias recientes en la Web of Science (WoS) (Martínez-Martínez; Aguado; Boeykens, 2017; Gonzálvez-Cabañas; Mochón; 2016; Chen, 2016) y sobre todo, el libro de Busch (2016) que engloba a varios autores y perspectivas.

Sin embargo, las empresas del sector que conviven con procesos programáticos son testigos de los cambios y se los están transmitiendo a las marcas para llegar a su público. Algunos anunciantes ya han tenido éxito en este entorno, como: Mapfre (Díaz, 2017) y L'Oréal (2016), premiado en el Il Foro de publicidad programática de 2016 (Blue 449, 2016). Otras grandes marcas como Mercedes Benz, HSBC, Disney, Dropbox y Thomson Reuters, también están confiando en esta clase de publicidad (Dircomfidencial, 2017).

En cualquier caso, la premisa de partida más importante para entender el paso de la publicidad online tradicional a la programática ha sido que no podemos seguir peleando contra la saturación generando más contenidos para las mismas personas (sin segmentarlas eficazmente).

En esta afirmación hay dos aspectos fundamentales a tener en cuenta:

\section{- la importancia de los contenidos;}

- el tratamiento de datos que permite una mejor segmentación de perfiles de audiencias.

En cuanto a los contenidos de los anuncios en publicidad programática, lo conveniente no es generar más volumen de anuncios para todos los usuarios, sino hacer llegar un mensaje diferente en función de la segmentación de audiencia.

Las formas de hacerlo se están diversificando, destacando el uso de la publicidad nativa o content advertising, que relaciona el contenido no publicitario de una web que ha generado interés en una persona, con otro contenido publicitario en donde una marca continúa el relato manteniendo el formato y el estilo iniciales (Tomas, 2017). 
Tabla 1. Diferencias entre el ecosistema de publicidad online tradicional y el ecosistema programático

\begin{tabular}{|c|c|}
\hline Ecosistema de publicidad online tradicional & Ecosistema de publicidad programática \\
\hline $\begin{array}{l}\text { Se basa en que el anunciante compra el espacio donde aparecerá su } \\
\text { anuncio, según el tipo de usuarios que entren en una web. }\end{array}$ & $\begin{array}{l}\text { El anunciante compra el espacio publicitario en función de qué usuario vaya } \\
\text { a ver el anuncio en un momento concreto. }\end{array}$ \\
\hline La estrategia es pull. Atraer a la audiencia hacia mi contenido. & La estrategia es push. Llevar el contenido personalizado hacia la audiencia. \\
\hline $\begin{array}{l}\text { Los anunciantes (o las agencias de medios en su nombre) eligen } \\
\text { dónde aparecerán los anuncios. }\end{array}$ & No se elige dónde colocar los anuncios sino a quién enseñárselos. \\
\hline $\begin{array}{l}\text { Un anunciante puede estudiar la audiencia y crear una estrategia } \\
\text { en base a su público objetivo, pero todos los impactos lanzados no } \\
\text { serán efectivos. }\end{array}$ & $\begin{array}{l}\text { Hace posible el impacto único de un anuncio por usuario. De esta forma se } \\
\text { garantiza a priori que todos los impactos serán útiles. }\end{array}$ \\
\hline $\begin{array}{l}\text { Los datos son fundamentales, pero tenerlos no aporta una ventaja } \\
\text { competitiva. }\end{array}$ & $\begin{array}{l}\text { Garantiza la utilidad de los datos, lo que se convierte en una ventaja compe- } \\
\text { titiva para las empresas. }\end{array}$ \\
\hline $\begin{array}{l}\text { Los anuncios se comercializan a CPM (coste por mil impresiones), } \\
\text { CPC (coste por click), CPA (coste por adquisición) o CPL (coste por } \\
\text { lead/registro). Todos se contratan a la vez y a precios fijos. }\end{array}$ & $\begin{array}{l}\text { Los anuncios se comercializan a CPM, pero se pagan uno a uno, en tiempo } \\
\text { real y con precios diferentes. }\end{array}$ \\
\hline $\begin{array}{l}\text { Los formatos pueden ser audio, vídeo y display (el más conocido, el } \\
\text { banner). Existen múltiples posibilidades de combinar los tres (rich } \\
\text { media). }\end{array}$ & $\begin{array}{l}\text { Los formatos pueden ser el display (tipo banner o texto) y el vídeo. Según } \\
\text { MarketingNews (2016), Spotify (plataforma de música por internet) comienza } \\
\text { a ofrecer en programática el formato audio. }\end{array}$ \\
\hline
\end{tabular}

Así, aun aumentando el contenido, no hay saturación puesto que en la fase de prospección de las posibles audiencias, después de una serie de impactos iniciales, si el usuario no interactúa se deja de comprar ese perfil y el anuncio no se genera.

En cuanto al tratamiento de datos, se favorece la segmentación, convirtiendo los datos en información útil que permita a un usuario concreto recibir sólo lo que necesita en cada momento y al anunciante pagar sólo por los impactos que le interesan (Lasheras et al., 2014).

\section{Claves para entender la publicidad programática y sus retos futuros}

Aunque la publicidad programática aparece ante la necesidad de simplificar los procesos tradicionales, lo cierto es que encierra gran complejidad (Sevillano-Zabala, 2015) y algunos problemas por resolver.

El proceso se lleva a cabo a través de una serie de players o agentes fundamentales (IAB, 2014).

El primer agente es el anunciante que demanda y compra impresiones de publicidad digital a través de una agencia de medios. El anunciante cuenta con la ayuda de plataformas automatizadas de compra o demand side platform (DSP) que adquieren impresiones o posibilidad de captar a un usuario para el anunciante.

Por otra parte están los soportes que ofrecen sus espacios publicitarios (publishers) a través de la plataforma de oferta o supply side platform (SSP). EI SSP es el medio que utilizan los soportes para poner en venta su inventario. Existen además redes publicitarias o ad networks que se encargan de almacenar y gestionar a la vez todo el inventario perteneciente a varios soportes digitales, ofreciendo segmentación de audiencia personalizada para cada anunciante.

Las plataformas de compra y venta actúan en el mercado virtual, el ad exchange:

- el ad exchange abierto permite que todos los anunciantes y soportes participen en cualquier subasta;
- el ad exchange privado sólo permite participar a ciertos anunciantes y soportes por acuerdo previo.

En el ad exchange se ofrece una impresión (posibilidad de captar a un usuario) en tiempo real, preguntando a qué precio quieren comprarla los anunciantes y a qué precio quieren venderla los soportes que poseen en su inventario la ubicación para llegar a ese usuario (Münstermann, Würtenberger, 2016). En este momento entran en juego las reglas fijadas a priori, decidiendo el precio de dicha impresión y atribuyendo la impresión al anunciante que más alto puja.

En resumen, todo el proceso se puede simplificar diciendo que un usuario navega por una web. Esta web aloja una cookie que se descarga en el navegador del usuario cuando accede a distintas páginas web y recoge la información de su navegación, lo que permite crear un perfil de ese usuario (data).

El anunciante compra el espacio publicitario en función de qué usuario vaya a ver el anuncio en un momento concreto

Por su parte los ad servers se encargan de almacenar dicha información. A través ves de un DMP (data management platform) se estructuran los datos recogidos y se distribuyen en segmentos de audiencia (iProspect, 2016). En base a esta información se decide si es rentable impactar a un sujeto y entrar en la subasta en tiempo real con otras plataformas de compra. Si la decisión es no, es porque el tratamiento de los datos ha permitido saber que el usuario no es considerado público objetivo, no perdiendo el dinero que se hubiese pagado por el impacto.

Una vez aclarado el proceso de la publicidad programática y los elementos que la determinan (Rodríguez-Silgado, 2017), se profundiza en la aportación de los profesionales para establecer los retos que van a definir su futuro y que se refieren a cada uno de sus factores clave:

- el factor humano; 
- perfiles de audiencias;

- tecnologías;

- automatización de los procesos.

Cada uno de estos factores clave depende de los otros y se explica mediante su presencia conjunta en este entorno. Esto dificulta en parte la tarea de los profesionales para precisar los retos por alcanzar y complica la búsqueda de soluciones.

Para resolver el problema de la saturación hay que conectar los contenidos con los individuos, uno a uno y en tiempo real

\section{Factor audiencia}

La publicidad programática forma parte de un ecosistema donde los datos son imprescindibles para la efectividad de la estrategia publicitaria digital. En el mercado programático ya no se compran espacios sino perfiles de audiencias. Por tanto, de los datos masivos (big data) o sistemas que gestionan grandes conjuntos de datos (Serrano-Cobos, 2014, p. 565), sólo interesan los que contengan una información útil para el anunciante (Fernández-Manzano; Neira; Clares-Gavilán, 2016) y le ayuden en la definición de su objetivo de campaña y del público objetivo a alcanzar.

En programática, según Lotame Solutions (2013), se utilizan varios tipos de datos de forma combinada:

- first party data: o datos de primera mano, propiedad del anunciante;

- second party data: aquellos que aun sabiendo exactamente su procedencia, proporcionan menos información y peor segmentación de usuarios;

- third party data: datos de terceros cuya procedencia se desconoce pero que el anunciante compra para realizar una mejor segmentación e impactar de forma más efectiva.

La gestión de estos datos permitirá por el lado de la venta optimizar el conjunto de sitios web disponibles y clasificados en base a la información que se maneja sobre los usuarios (el inventario), para adecuarlo a las necesidades de los anunciantes. Por el lado de la compra permitirá seleccionar cada vez mejor los perfiles que cada anunciante necesita.

No obstante, es importante saber que la calidad de los datos que han sido usados para la segmentación y compra del inventario de sitios web es proporcional a la respuesta de la audiencia ante los impactos publicitarios. Así, el precio de compra de los datos de audiencia aumenta a medida que se ofrece más exclusividad a un anunciante frente a otros.

En cualquier caso, un reto fundamental es aclarar los factores que definen las características de los datos de audiencia y que determinan su calidad y su precio. Los más importantes son:

- visibilidad del anuncio por parte de la audiencia específica;

- el precio y la compra de datos a terceros;

- exclusividad para unos anunciantes frente a otros a la hora de acceder a un inventario de sitios web;

- forma de identificar a los usuarios desde las distintas plataformas tecnológicas.

De esto se desprende a su vez el principal problema por resolver, la necesidad de garantizar la transparencia en relación con la información de audiencias y los sitios donde se ha mostrado el anuncio, que tanto el anunciante como la agencia de medios deberían conocer (Sáez, 2014). De momento no se asegura la transparencia informativa ni en los mercados públicos ni en los privados. La transparencia será pues un reto mientras el flujo de información entre el soporte, la agencia de medios y el anunciante oculte determinados datos.

En publicidad programática no se elige dónde colocar los anuncios sino a quién enseñárselos

\section{Factor tecnológico}

Se puede decir que la tecnología es lo que diferencia la compra y venta de publicidad digital de la publicidad programática. La tecnología permite que la publicidad programática sea personalizada en tiempo real, ofreciendo a cada usuario lo que espera y optimizando la inversión de los anunciantes.

Aunque más que un reto por resolver es una circunstancia fruto de la evolución actual de la publicidad programática, es fundamental detenerse en el hecho de que la publicidad programática no siempre se compra en tiempo real. La compra en tiempo real o RTB (real time bidding) nace ante la gran oferta de espacios en internet y la demanda de los anunciantes por colocar sus anuncios en el sitio adecuado, lo que provoca la aparición de un sistema de subasta de es-

Tabla 2. Comparación de RTB y publicidad programática

\begin{tabular}{|l|l|}
\hline \multicolumn{1}{|c|}{ Real time bidding (RTB) } & \multicolumn{1}{c|}{ Publicidad programática } \\
\hline $\begin{array}{l}\text { Compra y venta de impactos publicitarios exclusivamente en } \\
\text { tiempo real. }\end{array}$ & $\begin{array}{l}\text { Incluye la compra y venta en tiempo real, pero también puede haber acuerdos } \\
\text { no en tiempo real. }\end{array}$ \\
\hline Compra y venta online, exclusivamente basado en pujas. & Incluye la compra y venta en la subasta o mediante acuerdos privados previos. \\
\hline Se centra en la acción de comprar y vender. & $\begin{array}{l}\text { En programática se incluye el tratamiento de datos, antes y durante la subasta } \\
\text { en tiempo real. Se cruzan datos de audiencias para pujar sólo por los espacios } \\
\text { afines a un perfil de usuario. }\end{array}$ \\
\hline $\begin{array}{l}\text { El precio se determina en la subasta, en milésimas de segundo y y } \\
\text { es diferente para cada impresión. }\end{array}$ & $\begin{array}{l}\text { El precio se establece en tiempo real en la subasta, pero también se puede } \\
\text { comprar a precio fijo, sin subasta. }\end{array}$ \\
\hline $\begin{array}{l}\text { En el momento del RTB no existe intervención humana, sólo } \\
\text { interviene la tecnología. }\end{array}$ & $\begin{array}{l}\text { En programática la toma de decisiones conlleva gran gestión por parte del } \\
\text { equipo humano. }\end{array}$ \\
\hline
\end{tabular}


Tabla 3. Presencia del factor tecnológico y humano en el proceso programático

\begin{tabular}{|c|c|c|c|c|}
\hline $\begin{array}{c}\text { Fases } \\
\text { (funnel) }\end{array}$ & Acciones & $\begin{array}{c}\text { Tecnología/ } \\
\text { automatización }\end{array}$ & Factor humano & Nuevos perfiles \\
\hline \multirow{3}{*}{$\begin{array}{l}\text { Prospección. } \\
\text { Fase inicial en la que se aborda } \\
\text { a usuarios concretos con dife- } \\
\text { rentes segmentaciones pero, a } \\
\text { priori, sin excluir a nadie }\end{array}$} & $\begin{array}{l}\text { Elección del público } \\
\text { objetivo }\end{array}$ & Análisis de datos & Interpretar los datos & \multirow{3}{*}{$\begin{array}{l}\text { Media trader. Trading desk } \\
\text { Son profesionales de la información } \\
\text { encargados de convertir los datos } \\
\text { en útiles. Analizan los objetivos del } \\
\text { anunciante. Manejan una o varias pla- } \\
\text { taformas de compra de impactos con } \\
\text { la finalidad de distribuir el presupues- } \\
\text { to. Establecen las reglas de referencia } \\
\text { en la subasta }\end{array}$} \\
\hline & $\begin{array}{l}\text { Identificación previa } \\
\text { del target }\end{array}$ & $\begin{array}{l}\text { Rastreo de los movi- } \\
\text { mientos de usuarios }\end{array}$ & $\begin{array}{l}\text { Análisis de los momen- } \\
\text { tos de actividad de los } \\
\text { usuarios }\end{array}$ & \\
\hline & $\begin{array}{l}\text { Negociación de } \\
\text { acuerdos privados }\end{array}$ & $\begin{array}{l}\text { Mercados abiertos o } \\
\text { privados }\end{array}$ & $\begin{array}{l}\text { Elección de acuerdos y } \\
\text { reglas }\end{array}$ & \\
\hline $\begin{array}{l}\text { Consideración. } \\
\text { Se selecciona a los usuarios que } \\
\text { han interactuado con la marca }\end{array}$ & $\begin{array}{l}\text { Elección del target } \\
\text { óptimo }\end{array}$ & $\begin{array}{l}\text { Identificación de los } \\
\text { perfiles óptimos }\end{array}$ & $\begin{array}{l}\text { Selección de los perfiles } \\
\text { finales }\end{array}$ & \multirow{3}{*}{$\begin{array}{l}\text { Programmatic media planner. } \\
\text { Planifica la estrategia de la campaña, } \\
\text { señala los perfiles de audiencia que } \\
\text { quiere segmentar/comprar y lo comu- } \\
\text { nica al trader }\end{array}$} \\
\hline \multirow{2}{*}{$\begin{array}{l}\text { Acción. } \\
\text { Se combina la información so- } \\
\text { bre recencia, usuarios recientes, } \\
\text { y la frecuencia, abordando a } \\
\text { aquellos que han empezado el } \\
\text { proceso, una o varias veces y, o } \\
\text { bien no lo han terminado o lo } \\
\text { hicieron hace tiempo. Se juega } \\
\text { cruzando las variables }\end{array}$} & $\begin{array}{l}\text { Creación de estrate- } \\
\text { gias por públicos }\end{array}$ & $\begin{array}{l}\text { Optimiza la estrategia } \\
\text { y permite llegar a } \\
\text { diferentes públicos } \\
\text { a la vez }\end{array}$ & $\begin{array}{l}\text { Selección de perfiles } \\
\text { segmentados para de- } \\
\text { sarrollar las estrategias } \\
\text { previstas }\end{array}$ & \\
\hline & Diseño creativo & $\begin{array}{l}\text { Diseño de creativida- } \\
\text { des dinámicas que } \\
\text { cambian en función } \\
\text { de quien lo ve }\end{array}$ & $\begin{array}{l}\text { Diseñadores gráficos } \\
\text { y creativos deciden las } \\
\text { creatividades y formas } \\
\text { de abordar al usuario }\end{array}$ & \\
\hline Evaluación & Análisis de resultados & $\begin{array}{l}\text { Obtener resultados } \\
\text { en tiempo real }\end{array}$ & $\begin{array}{l}\text { Supervisar la consecu- } \\
\text { ción de objetivos para } \\
\text { campañas futuras o } \\
\text { para obtener resultados } \\
\text { finales }\end{array}$ & $\begin{array}{l}\text { Data scientist. Data intelligence } \\
\text { Trabajan sólo con datos. Miden y ana- } \\
\text { lizan los resultados y dan soluciones } \\
\text { para nuevas campañas. También son } \\
\text { útiles en otras partes del proceso } \\
\text { (funnel) para saber de dónde proce- } \\
\text { den exactamente las interacciones } \\
\text { del usuario (qué anuncio le ha hecho } \\
\text { comprar o llegar a una web) }\end{array}$ \\
\hline
\end{tabular}

Elaborado a partir de Macías (2017) e IPMark (2017)

pacios por los que se puja, pagando un precio diferente por cada impacto conseguido en tiempo real.

La publicidad programática es diferente del RTB (tabla 2) y por tanto, no siempre que hablamos de programática nos referimos a RTB. Aunque toda compra en tiempo real es compra programática, no toda la publicidad programática se compra en tiempo real (Rodríguez- Silgado, 2017).

El modelo de compra más utilizado en publicidad programática es el RTB, pero también es posible la compra por acuerdos previos y a precio fijo en mercados privados (IAB, 2014). Aunque un acuerdo en un mercado programático privado no suceda en tiempo real y por tanto no se pueda considerar RTB, sí es publicidad programática, ya que la compra y venta de los espacios va ligada a los datos de audiencia y a las tecnologías programáticas. En resumen, en publicidad programática intervienen varios modelos de negocio, en tiempo real o no, que se pueden combinar (Chen; Shuai; Wang, 2015).

En tiempo real o no, un reto tecnológico fundamental en programática es mejorar las estrategias cross-device. El uso de diferentes dispositivos con acceso a internet ha creado la necesidad de identificar al usuario independientemente del tipo de dispositivo que se use. Se puede reconocer a un usuario a través de un identificador común (ID) utilizado para señalar que el usuario es el mismo en los diferentes dispositivos, garantizando así la calidad de los impactos y optimizando el presupuesto de los anunciantes.
La tecnología también deberá asumir el reto de permitir el uso único del vCPM (coste por mil impresiones visibles) frente al tradicional modelo de compra CPM (coste por mil impresiones) actualmente utilizado en las comercializaciones y acuerdos programáticos (tabla 1). La diferencia es esencial, ya que para quien realiza la acción de compra de impresiones es preferible pagar sólo por aquellas que sean vistas por el usuario (pagar por visibilidad, viewability).

Por último, un reto tecnológico muy importante es garantizar un entorno web seguro para todos los players. En programática un entorno web seguro se puede entender como aquel en el que:

- sea posible controlar dónde aparecen los anuncios: muchos anunciantes no quieren relacionarse con algunas webs o viceversa (el anunciante debe saber exactamente dónde compra y dónde ha aparecido);

- a quién está impactando.

A día de hoy la publicidad programática sólo puede garantizar un entorno seguro a través de acuerdos privados o en el mercado programático abierto si utiliza white o black lists. Esto no ha funcionado bien en todos los casos, y se han generado situaciones incómodas en donde, por ejemplo, la publicidad de marcas como Mercedes-Benz se ha visto insertada en vídeos terroristas (Dircomfidencial, 2017), con gran perjuicio para la reputación de estas empresas. 


\section{Factor humano frente a automatización}

Aunque la automatización de los datos es necesaria y simplifica los procesos, el equipo humano es también fundamental en todas las acciones que conlleva cada una de las fases de compra (funnel): prospección, consideración, acción y evaluación (tabla 3).

La presencia del factor humano se garantiza en todo el proceso por el diseño de nuevas funciones. El reto es que los nuevos profesionales no sólo deberán tener conocimientos de marketing y comunicación sino también de programación y gestión de datos. Dejando al margen la aparición de perfiles relacionados directamente con la informática o herederos de los perfiles tradicionales (IpMark, 2017), están apareciendo otros relacionados con las ciencias de la información (tabla 3).

\section{Se convierten los datos en información útil, que permita a un usuario concreto recibir sólo lo que necesita en cada mo- mento y al anunciante pagar sólo por los impactos que le interesan}

\section{Conclusión}

La publicidad programática no es un conjunto de nuevos formatos en publicidad digital. Se considera un cambio en la comercialización de dicha publicidad que transcurre paralelo al desarrollo de la tecnología y que es posible, a partes iguales, gracias a la gestión de datos de audiencias, la automatización de procesos y la participación del factor humano.

A priori, la gestión de datos en publicidad programática aporta grandes ventajas a todos los agentes implicados en el proceso. Los anunciantes podrán comprar las impresiones que les interesan en el mismo momento en que un usuario está visitando la web, decidiendo en tiempo real si se considera público objetivo o no y asegurando su inversión. Los soportes que venden sus espacios cuentan también con la gestión de datos para generar nuevas oportunidades de optimizar sus inventarios, dependiendo de la demanda real existente. El usuario se ve también afectado positivamente: la publicidad será más relevante y de mayor interés para él, resolviendo el desinterés por la excesiva oferta de anuncios que interesan a pocas personas.

Por tanto, las ventajas que ofrece la publicidad programática están relacionadas con simplificar la comercialización y generar una publicidad no intrusiva, disminuyendo la saturación publicitaria. Así, aumentará el retorno de la inversión (ROI) para los anunciantes y los soportes sacarán mayor provecho a sus espacios en internet.

No obstante, los principales retos a alcanzar se centran en la necesidad de:

- perfeccionar y abaratar los procesos, para garantizar la ubicación de las audiencias en diferentes soportes;

- transparencia de los resultados obtenidos frente al cliente;

- pagar sólo por la visibilidad (viewability) obtenida (López, 2017).
El ecosistema programático debe mantener el equilibrio entre la automatización de los procesos y el factor humano. En esta batalla hombre-máquina el problema se convierte en una oportunidad para crear nuevos perfiles profesionales. Serán necesarias la interdisciplinariedad entre las ciencias de la información y la comunicación, y la especialización en procesos de gestión de datos específicos. Las empresas de marketing/publicidad y agencias de medios necesitan nuevos perfiles orientados a la informática, las matemáticas y la estadística, pero sobre todo se busca un profesional de la comunicación digital que sea un híbrido entre analista de datos y estratega, lo que se reúne en nuevos perfiles vinculados a las ciencias de la información, tales como media trader, programmatic media planner, data scientist y data intelligence.

En definitiva, asumiendo que el factor tecnológico es indispensable para alcanzar los retos, el problema real no está siendo la tecnología, sino el precio de las herramientas y trabajos creativos que la hacen posible. El coste es más elevado que si se impacta a todos los usuarios potenciales con el mismo anuncio. Por el momento los anunciantes siguen reacios a pagar por esta mejora en la forma de llegar a su público, lo que a su vez dificulta la solución de los problemas detectados.

Por último, si se superan los retos y se consigue extender su campo de actuación a otros medios, no sólo a internet, la publicidad programática se volverá útil para el público y rentable para los agentes y áreas de conocimiento que intervienen.

Los nuevos profesionales no sólo deberán tener conocimientos de marketing y comunicación sino también de programación y gestión de datos

\section{Referencias}

Aguado, Juan-Miguel; Martínez-Martínez, Inmaculada J.; Cañete-Sanz, Laura (2015). "Tendencias evolutivas del contenido digital en aplicaciones móviles". El profesional de la información, v. 24, n. 6, pp. 787-795.

https://doi.org/10.3145/epi.2015.nov.10

Bahamonde, Elsa (2016). "La publicidad programática no vende patitos feos". Anuncios: Semanario de publicidad y marketing, n. 1537, 26 septiembre.

https://goo.gl/c8sMuR

Blue 449 (2016). "L’Oréal y Optimedia ganan el Gran premio de publicidad programática". Intelligence. Noticias y estudios del sector de la comunicación. https://goo.gl/Y3LZqz

Busch, Oliver (ed.) (2016). Programmatic advertising. Berlin: Springer International Publishing. ISBN: 9783319250236 https://doi.org/10.1007/978-3-319-25023-6

Chen, Bowei (2016). "Risk-aware dynamic reserve prices of programmatic guarantee in display advertising". In: Data mining workshops (Icdmw), $16^{\text {th }}$ Intl conf of IEEE, pp. 511-518. 
https://goo.gl/HBC8Fx

https://doi.org/10.1109/ICDMW.2016.0079

Chen, Bowei; Shuai, Yuan; Jun, Wang (2014). "A dynamic pricing model for unifying programmatic guarantee and real-time bidding in display advertising". In: Procs of the $8^{\text {th }}$ IntI workshop on data mining for online advertising, pp. 1-9, ACM. http://yuan-shuai.info/paper/ADKDD-2014-unifyingprogrammatic-guarantee.pdf

Díaz, Jesús (2017). “\#Programatica. Cómo pasar a programática a un cliente de gran consumo y no morir en el intento". El programa de la publicidad de Jesús Díaz, 14 febrero. https://goo.gl/8YWmfB

Dircomfidencial (2017). "Las marcas culpan a la publicidad programática por la vinculación de sus anuncios a contenidos terroristas". Dircomfidencial, 15 febrero.

https://goo.gl/MNf3FP

Fernández-Manzano, Eva-Patricia; Neira, Elena; Clares-Gavilán, Judith (2016). "Data management in audiovisual business: Netflix as a case study". El profesional de la información, v. 25, n. 4, pp. 568-576.

https://doi.org/10.3145/epi.2016.jul.06

Gilardoni, Claudia (2013). “La información ¿es poder?”. Infotecarios, 5 febrero.

http://www.infotecarios.com/la-informacion-es-poder

Gonzálvez-Cabañas, Juan-Carlos; Mochón, Francisco (2016). "Operating an advertising programmatic buying platform: A case study". Intl journal of interactive multimedia \& artificial intelligence, v. 3, n. 6, pp. 6-15.

https://goo.gl/5dmJ2S

https://doi.org/10.9781/ijimai.2016.361

IAB (2014). Libro blanco de compra programática. Interactive Advertising Bureau.

https://goo.gl/yRt51n

IPMark (2017). "Los 12 perfiles imprescindibles en publicidad programática". IPMark, 10 julio.

http://ipmark.com/marketing-digital-programatica-empleos

iProspect (2016). “Compra programática y RTB para principiantes". iProspect,

https://www.iprospect.com/es/es/blog/compraprogramatica-y-rtb-para-principiantes

Lasheras, Chechu; Del-Olmo, Enrique; Recuenco, Javier; Valverde-Cohén, Jaime; Martín, Luis (2014). “Presente y futuro del márketing digital de resultados de los 'banners' a la compra programática". Harvard Deusto márketing y ventas, n. 128 , pp. 20-25.

https://goo.gl/vWCyEd

López, Ana (2017). “Fraude, transparencia y visibilidad: 3 tópicos que darán mucho de qué hablar en 2017 para la publicidad digital. Adsmóvil. Soluciones de publicidad móvil, 13 febrero.

https://goo.gl/SmUxuj
Lotame Solutions (2013). " $1^{\text {st }}$ party data, $2^{\text {nd }}$ party data, $3^{\text {rd }}$ party data: What does it all mean?". Lotame, April $30^{\text {th }}$. https://www.lotame.com/resource/1st-party-2nd-party3rd-party-data-what-does-it-all-mean

Macías, Raúl (2017). “El marketing programático ayuda a las marcas a aprovechar micro momentos". Actualidad, 20 septiembre.

https://www.tooltyp.com/marketing-programatico-ayudalas-marcas-aprovechar-micro-momentos

Martínez-Martínez, Inmaculada J.; Aguado, Juan-Miguel; Boeykens, Yannick (2017). "Ethical implications of digital advertising automation: The case of programmatic advertising in Spain". El profesional de la información, v. 26, n. 2, pp. 201-210.

https://doi.org/10.3145/epi.2017.mar.06

Martínez-Martínez, Silvia; Lara-Navarra, Pablo (2015). “EI big data transforma la interpretación de los medios sociales". El profesional de la información, v. 23, n. 6, pp. 575-581. http://dx.doi.org/10.3145/epi.2014.nov.03

Münstermann, Holm; Würtenberger, Peter (2016). "Programmatic disruption for premium publishers". En: Busch, O. (ed.), Programmatic advertising, management for professionals. Springer Switzerland, pp. 25-36. ISBN: 9783319 250212

https://goo.gl/i9vaFH

http://dx.doi.org/10.1007/978-3-319-25023-6_3

Rodríguez-Silgado, Ana (2017). La publicidad programática. El futuro de la publicidad digital. Trabajo fin de grado. Universidad de Extremadura.

http://hdl.handle.net/10662/5316

Sáez, Maite (2014). "RTB o el algoritmo que pretende cambiar la compra de publicidad. Un $20 \%$ de la inversión publicitaria podría moverse a través de la compra programática este año, según IAB Spain". Anuncios: Semanario de publicidad y marketing, n. 1487, pp. 42-44.

Selva-Ruiz, David; Caro-Castaño, Lucía (2016). "Uso de datos en creatividad publicitaria: el caso de Art, Copy \& Code de Google". El profesional de la información, v. 25, n. 4, pp. 642-651.

https://doi.org/10.3145/epi.2016.jul.14

Serrano-Cobos, Jorge (2014). "Big data y analítica web. Estudiar las corrientes y pescar en un océano de datos". El profesional de la información, v. 23, n. 6, pp. 561-565.

http://dx.doi.org/10.3145/epi.2014.nov.01

Sevillano-Zabala, Juan (2015). "Comprando usuarios y no medios. Dos puntos de vista sobre compra programática". Anuncios: Semanario de publicidad y marketing, n. 1515, p. 8.

Tomás, David (2017). “¿Qué es la publicidad nativa? Ventajas y casos de éxito". Cyberclick.

http://www.cyberclick.es/que-es-la-publicidad-nativaventajas-y-casos-de-exito 